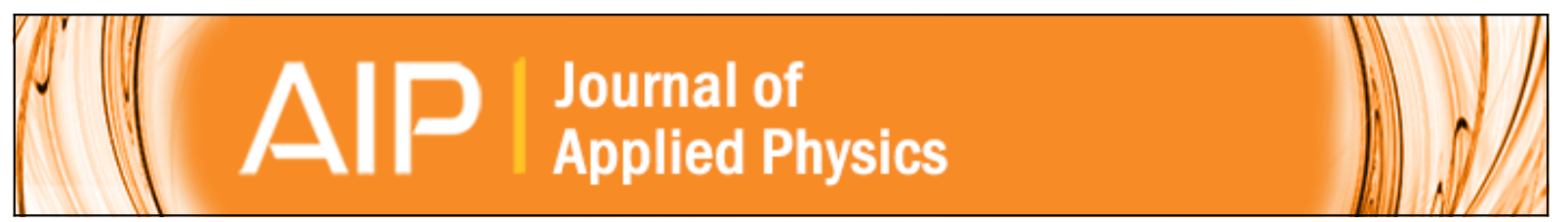

\title{
Magnetocaloric effect and critical exponents of Fe77Co5.5Ni5.5Zr7B4Cu1: A detailed study
}

V. Franco, R. Caballero-Flores, A. Conde, K. E. Knipling, and M. A. Willard

Citation: Journal of Applied Physics 109, 07 A905 (2011); doi: 10.1063/1.3535191

View online: http://dx.doi.org/10.1063/1.3535191

View Table of Contents: http://scitation.aip.org/content/aip/journal/jap/109/7?ver=pdfcov

Published by the AIP Publishing

\section{Articles you may be interested in}

Nanocrystalline Fe88-2 x Co x Ni x Zr7B4Cu1 alloys: Soft magnets for vehicle electrification technologies (invited)

J. Appl. Phys. 117, 172611 (2015); 10.1063/1.4914118

Magnetocaloric effect in Fe-Zr-B-M ( $\mathrm{M}=\mathrm{Ni}$, Co, $\mathrm{Al}$, and Ti) amorphous alloys

J. Appl. Phys. 116, 093910 (2014); 10.1063/1.4895048

Influence of $\mathrm{Co}$ and $\mathrm{Ni}$ addition on the magnetocaloric effect in Fe $88-2 \times \mathrm{Co} \times \mathrm{Ni} \times \mathrm{Zr} 7 \mathrm{~B} 4 \mathrm{Cu} 1$ soft magnetic amorphous alloys

Appl. Phys. Lett. 96, 182506 (2010); 10.1063/1.3427439

Cryogenic hysteretic loss analysis for (Fe,Co,Ni)-Zr-B-Cu nanocrystalline soft magnetic alloys

J. Appl. Phys. 101, 09N113 (2007); 10.1063/1.2714187

Structure and magnetic properties of nanocrystalline ( Fe 1-x Co x ) $90 \mathrm{Zr} 7 \mathrm{~B} 2 \mathrm{Cu} 1$ (0x0.6)

Appl. Phys. Lett. 76, 2110 (2000); 10.1063/1.126270

\section{SHIMADZU Powerful, Multi-functional UV-Vis-NIR and Excellence in science FTIR Spectrophotometers}

Providing the utmost in sensitivity, accuracy and resolution for applications in materials characterization and nano research

- Photovoltaics - Ceramics

- Polymers

- Thin films

- Paints

- DNA film structures

- Coatings

- Packaging materials
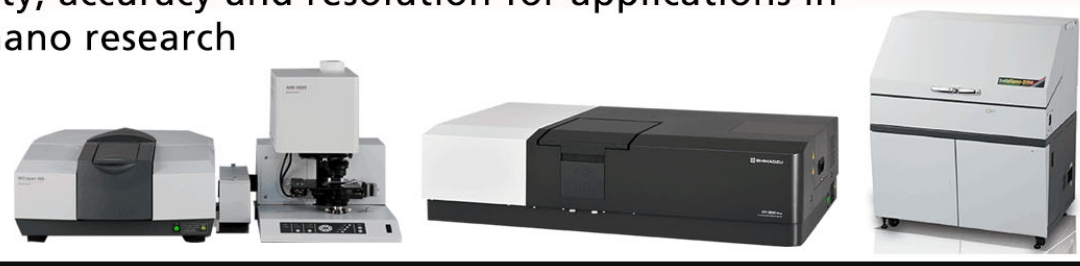


\title{
Magnetocaloric effect and critical exponents of $\mathrm{Fe}_{77} \mathrm{Co}_{5.5} \mathrm{Ni}_{5.5} \mathrm{Zr}_{7} \mathrm{~B}_{4} \mathrm{Cu}_{1}$ : A detailed study
}

\author{
V. Franco, ${ }^{1, a)}$ R. Caballero-Flores, ${ }^{1}$ A. Conde, ${ }^{1}$ K. E. Knipling, ${ }^{2}$ and M. A. Willard ${ }^{2}$ \\ ${ }^{1}$ Dpto. Física de la Materia Condensada, ICMSE-CSIC, Universidad de Sevilla, P.O. Box 1065, 41080 Sevilla, \\ Spain \\ ${ }^{2}$ Multifunctional Materials Branch, U.S. Naval Research Laboratory, 4555 Overlook Ave. SW, Washington, \\ District of Columbia 20375, USA
}

(Presented 15 November 2010; received 3 September 2010; accepted 6 October 2010; published online 21 March 2011)

\begin{abstract}
The critical exponents of the alloy have been determined with the Kouvel-Fisher method to predict the field dependence of the magnetic entropy change $\Delta S_{M}$. The nonlinear fit of $\Delta S_{M}(H)$ to a power law provides a field exponent in perfect agreement with the predictions of the relevant scaling laws using the obtained critical exponent values. It is shown that possible discrepancies between these two methods for determining the field dependence of $\Delta S_{M}$ might arise due to a poor resolution in the temperature of the experiments. (C) 2011 American Institute of Physics. [doi:10.1063/1.3535191]
\end{abstract}

The magnetocaloric effect and magnetic refrigeration at room temperature are gaining increased scientific attention due to the larger energy efficiency of magnetic refrigeration and its smaller footprint on the environment when compared to conventional compression/expansion of gases. ${ }^{1}$ To be of broad use, magnetic refrigerators should operate at low or moderate magnetic fields (in the 1-2 $\mathrm{T}$ range). Therefore, it is important to properly describe the field dependence of the magnetic entropy change $\Delta S_{M}$. There has been recent work which correlate this field dependence with the critical exponents of the ferroparamagnetic transition of the material, ${ }^{2-4}$ which are now being applied to a variety of materials groups. ${ }^{5,6}$ And for those cases in which standard techniques (like the Kouvel-Fisher method ${ }^{7}$ ) do not work properly, scaling laws for the magnetic entropy change have been proposed as alternative methods for determining the critical exponents of a material. ${ }^{8}$ Moreover, the scaling laws and the universal curve for the magnetocaloric effect can also be used as an alternative to the Banerjee criterion ${ }^{9}$ to determine the order of the phase transition only from magnetization measurements. ${ }^{10}$ However, in some specific cases, experimental results of the field dependence of the magnetic entropy change seem to be inconsistent with the behavior predicted by the critical exponents. For example, in the case of $\mathrm{FeCoNiZrBCu}$ alloys, values of the exponent for field dependence of the peak magnetic entropy change can vary by $\sim 20 \%$ depending on the temperature steps used for the isothermal magnetization experiments when calculated either from the values of the critical exponents or from the nonlinear fitting of the peak magnetic entropy change versus field. To test the generality of the scaling laws for the magnetocaloric effect, it is relevant to analyze if these discrepancies are due to intrinsic properties of the materials under study or if they are just a consequence of the experimental conditions used (i.e., whether the data is collected near enough to the $T_{C}$ or with dense enough temperature increments).

\footnotetext{
${ }^{a)}$ Author to whom correspondence should be addressed. Electronic mail: vfranco@us.es.
}

The literature on magnetocaloric materials is mainly centered on crystalline rare-earth based alloys and compounds, ${ }^{11-13}$ although there is evidence that some transition metal based amorphous alloys can surpass the refrigerant capacity (RC) of those more expensive compounds. For example, it has been recently shown ${ }^{14}$ that the addition of Co and $\mathrm{Ni}$ to Nanoperm-type amorphous alloys can produce an $\mathrm{RC} \sim 40 \%$ larger than $\mathrm{Gd}_{5} \mathrm{Si}_{2} \mathrm{Ge}_{1.9} \mathrm{Fe}_{0.1}$ (Ref. 15) and $\sim 15 \%$ larger than the previously studied Fe-based amorphous alloys. ${ }^{16}$ Additionally, amorphous alloys possess enhanced electrical resistivity, corrosion resistance, good mechanical properties, and tunability of the Curie transition, which are beneficial for magnetic refrigeration and are beginning to garner the interest of the scientific community.

The aim of this work is to study in detail the field dependence of $\Delta S_{M}$ for the soft magnetic amorphous alloy $\mathrm{Fe}_{77} \mathrm{Co}_{5.5} \mathrm{Ni}_{5.5} \mathrm{Zr}_{7} \mathrm{~B}_{4} \mathrm{Cu}_{1}$ in a narrow temperature range around its Curie temperature in order to show that the possible discrepancies between the critical exponents obtained from the Kouvel-Fisher technique and from the analysis of the magnetocaloric response of the sample can be simply ascribed to a limited experimental resolution in temperature.

Amorphous ribbons of $\mathrm{Fe}_{77} \mathrm{Co}_{5.5} \mathrm{Ni}_{5.5} \mathrm{Zr}_{7} \mathrm{~B}_{4} \mathrm{Cu}_{1}$ (typically 2-3 mm wide and $\sim 20 \mu \mathrm{m}$ thick) were obtained by a melt-spinning technique. Further details about sample preparation, microstructure, and magnetic characterization are given elsewhere. ${ }^{17}$

The change in magnetic entropy caused by a variation in applied magnetic field has been obtained by the numerical approximation of

$$
\Delta S_{M}(T, \Delta H)=\mu_{0} \int_{H_{0}}^{H_{f}}\left(\frac{\partial M(T, H)}{\partial T}\right)_{H} d H,
$$

where $\Delta H=H_{f}-H_{0}$ is the magnetic field change, $\mu_{0}$ is the magnetic permeability of vacuum, and $M(T, H)$ is the magnetization of the material. The field and temperature dependence of magnetization $M(T, H)$ was measured by vibrating sample magnetometry. Magnetic field increments were $10 \mathrm{mT}$ up to $\mu_{0} H=100 \mathrm{mT}, 20 \mathrm{mT}$ up to $1 \mathrm{~T}$ and $50 \mathrm{mT}$ up to $1.5 \mathrm{~T}$, 
and temperature increments were $2 \mathrm{~K}$ from 450 up to $479 \mathrm{~K}$, $1 \mathrm{~K}$ up to $499 \mathrm{~K}$, and $2 \mathrm{~K}$ up to $520 \mathrm{~K}$.

In order to determine the critical exponents, the KouvelFisher ${ }^{7}$ method has been used. It consists of an iterative procedure in which the Arrott-Noakes plot [i.e., the plot of $M^{2.5}$ versus $\left.(H / M)^{0.75}\right]$ is constructed. From it, the values for $M_{0}(T)$ are computed from the intercepts of various isothermal magnetization vs field curves on the ordinate of the plot (for temperatures below the Curie temperature $T_{C}$ ). The intercept on the abscissa allows calculation of $\chi_{0}(T)$ (for temperatures above $T_{C}$ ). Once the $M_{0}(T)$ and $\chi_{0}(T)$ curves have been constructed, two additional parameters $X(T)$ and $Y(T)$ are determined by

$$
\begin{gathered}
X(T)=\chi_{0}^{-1}\left(d \chi_{0}^{-1} / d T\right)^{-1}=\left(T-T_{C}\right) / \gamma \\
Y(T)=M_{0}\left(d M_{0} / d T\right)^{-1}=\left(T-T_{C}\right) / \beta
\end{gathered}
$$

In the critical region, both $X(T)$ and $Y(T)$ should be linear with slopes which give the values of the critical exponents and intercepts with the temperature axis that correspond to the Curie temperature. The values of the critical exponents are refined using an iterative method: Using the critical exponents from Eqs. (2) and (3), a generalized Arrott-Noakes plot $\left[M^{1 / \beta}\right.$ vs $\left.(H / M)^{1 / \gamma}\right]$ is constructed and used to calculate new $M_{0}(T)$ and $\chi_{0}(T)$ curves, which are subsequently input into Eqs. (2) and (3), resulting in newer values for $\beta$ and $\gamma$. The procedure finishes when the desired convergence of the parameters is achieved. Figure 1 shows the final iteration step for the $\mathrm{Fe}_{77}$ $\mathrm{Co}_{5.5} \mathrm{Ni}_{5.5} \mathrm{Zr}_{7} \mathrm{~B}_{4} \mathrm{Cu}_{1}$ amorphous alloy. The values extracted from this plot are $\beta=0.53 \pm 0.03, \gamma=1.34 \pm 0.04$, and $T_{C}=493.3 \pm 0.3 \mathrm{~K}$. The scaling relation $\beta \delta=\beta+\gamma$ allows for the calculation of $\delta=3.5 \pm 0.4$.

The reliability of the obtained exponents and Curie temperatures can be ascertained by checking the scaling of the magnetization curves. For magnetic systems, the scaling equation of state takes the form ${ }^{18,19}$

$$
\frac{H}{M^{\delta}}=h\left(\frac{\varepsilon}{M^{1 / \beta}}\right)
$$

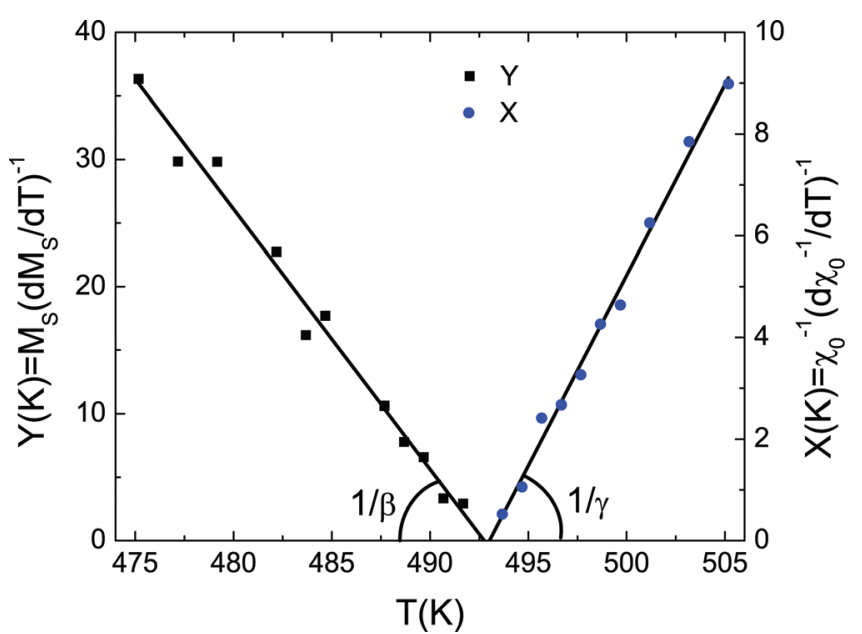

FIG. 1. (Color online) Determination of the critical exponents and Curie temperature for the $\mathrm{Fe}_{77} \mathrm{Co}_{5.5} \mathrm{Ni}_{5.5} \mathrm{Zr}_{7} \mathrm{~B}_{4} \mathrm{Cu}_{1}$ alloy using the Kouvel-Fisher method. where $\varepsilon=\left(T-T_{C}\right) / T_{C}$ is the reduced temperature, $h(x)$ is a scaling function, and $\beta$ and $\delta$ are critical exponents which characterize the magnetization behavior along coexistence $(H=0, \varepsilon<0)$ and the critical isotherm $(\varepsilon=0)$, respectively. Equation (4) may be formally inverted as

$$
\frac{M}{|\varepsilon|^{\beta}}=m_{ \pm}\left(\frac{H}{|\varepsilon|^{\Delta}}\right)
$$

where $\Delta=\beta \delta$ is the gap exponent and the plus (minus) sign corresponds to $\varepsilon>0 \quad(\varepsilon<0)$, respectively. Therefore, according to Eq. (4), if the appropriate values for the critical exponents and $T_{C}$ are used, the plot of $M / H^{1 / \delta}$ versus $\varepsilon / H^{1 / \Delta}$ (which corresponds to $1 / h^{1 / \delta}$ vs $x / h^{1 / \beta \delta}$, where $x=\varepsilon / M^{1 / \beta}$ ) should correspond to a universal curve onto which all experimental data points collapse. Alternatively, Eq. (5) indicates that $M /|\varepsilon|^{\beta}$ versus $H /|\varepsilon|^{\Delta}$ should result in two universal curves, one for $\varepsilon>0$ and the other for $\varepsilon<0$. These two constructions are plotted in Fig. 2, showing the reliability of the obtained values.

Taking into account that the field dependence of the magnetic entropy change can be expressed as a power law of the field ${ }^{2-4} \Delta S_{M}(T, H) \propto H^{n}$, with an exponent at $T=T_{C}$ which is $n=(1-\alpha) / \Delta$, and using Griffiths equality $(1-\alpha$ $=\beta+\Delta-1)$, these critical exponents predict $n=0.75$ \pm 0.03 .

Alternatively, the exponent $n$ can be obtained from the nonlinear power law fit at the temperature of the peak entropy change $\left(T_{\mathrm{pk}}\right)$ vs field. With the obtained experimental data, this procedure provides $n=0.756 \pm 0.003$, in perfect agreement with the values obtained from the critical exponents.

However, an apparently good fit is found if data collected at temperatures too far from the transition temperature are analyzed in this way. For instance, the data presented in Ref. ${ }^{11}$ have relatively coarse temperature steps resulting in a fit of the nonlinear region yielding $\Delta S_{M}\left(T=T_{\mathrm{pk}}-5 \mathrm{~K}\right)$, $n_{\text {app }} \sim 0.80$. It is worth noting that the only two temperatures

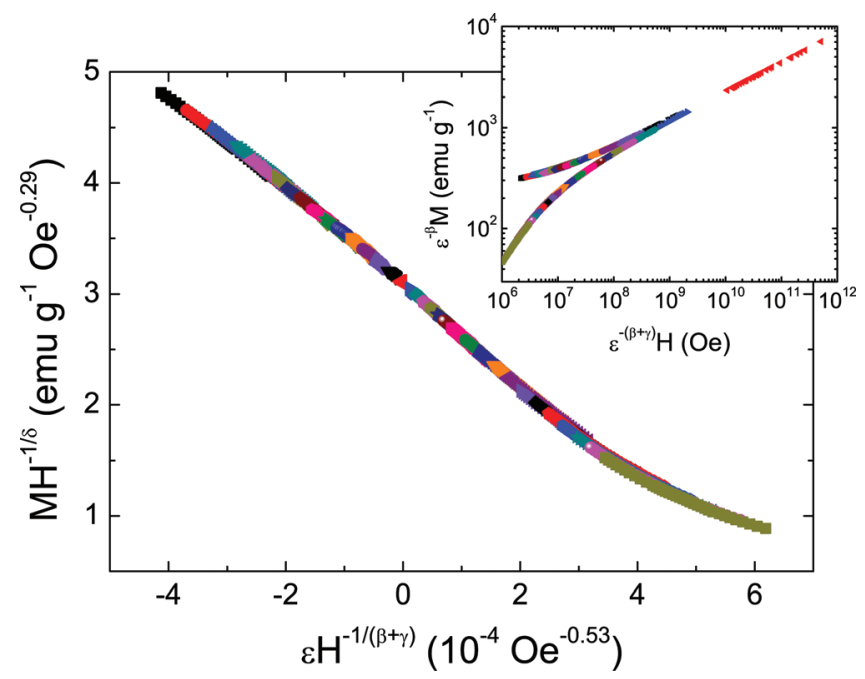

FIG. 2. (Color online) Two different scalings of the thermomagnetic curves to evidence the validity of the critical exponents and $T_{C}$ values obtained from the Kouvel-Fisher analysis. 


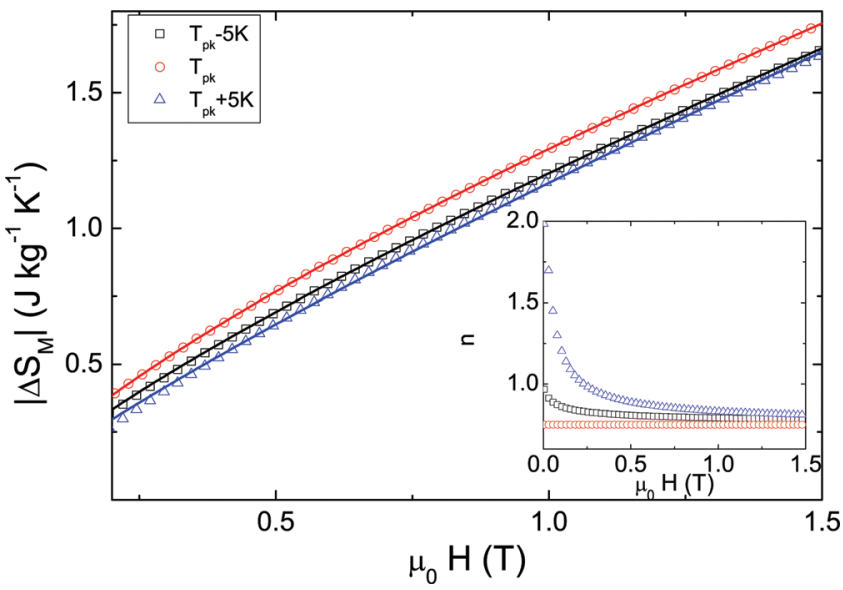

FIG. 3. (Color online) Field dependence of the magnetic entropy change of a material which follows the Arrott-Noakes equation of state with the same parameters of the $\mathrm{Fe}_{77} \mathrm{Co}_{5.5} \mathrm{Ni}_{5.5} \mathrm{Zr}_{7} \mathrm{~B}_{4} \mathrm{Cu}_{1}$ alloy. Lines are power law fits of the data, resulting in $n\left(T_{C}\right)=0.753$ (as predicted by the critical exponents), $n\left(T_{C}-5 \mathrm{~K}\right)=0.801$, and $n\left(T_{C}+5 \mathrm{~K}\right)=0.863$. Inset: field dependence of the locally calculated exponent $\mathrm{n}$ at the three indicated temperatures.

which provide a field independent value for $n$ are $T_{\mathrm{pk}}$ and $T_{C}{ }^{20}$ Therefore, making the fit at temperatures close to them might give an apparently good fit, but with an apparent exponent value larger than the actual one.

In order to evidence with numerical simulations that this explanation accounts for the experimentally observed discrepancies, the temperature and field dependent magnetization curves have to be modeled using an equation of state. It has recently been shown that the Arrott-Noakes equation of state ${ }^{21}$

$$
H^{1 / \gamma}=a\left(T-T_{C}\right) M^{1 / \gamma}+b M^{1 / \beta+1 / \gamma}
$$

can be used to represent the thermomagnetic response of soft magnetic amorphous alloys in the environment of their $T_{C}{ }^{22}$ The main panel of Fig. 2 has been used to obtain the parameters $a$ and $b$ from this equation of state by performing a nonlinear fit. The obtained parameters are $a=3.477 \pm 2$ $\times 10^{-3}(\mathrm{Oeg} / \mathrm{emu})^{1 / \gamma} \mathrm{K}^{-1}$ and $b=0.05323 \pm 3 \times 10^{-5} \mathrm{Oe}^{1 / \gamma}$ $(\mathrm{g} / \mathrm{emu})^{1 / \beta+1 / \gamma}$.

Figure 3 shows the numerically generated magnetic entropy change curves for a material whose equation of state is expressed by Eq. (6) and whose parameters are those obtained from fitting the data from the alloy studied in this paper. In this case, the nonlinear fit provides $n\left(T_{C}\right)=0.753$ (as predicted by the critical exponents), for $n\left(T_{C}-5 \mathrm{~K}\right)=0.801$ and
$n\left(T_{C}+5 \mathrm{~K}\right)=0.863$. The inset of Fig. 3 shows the field dependence of the locally calculated exponent $n$ for these three temperatures, indicating that the larger the applied field, the lower the separation from the proper value of $n$ at the critical temperature. This analysis confirms that the discrepancies which might appear when analyzing the field dependence of the magnetic entropy change either by the critical exponents or by nonlinear fits of the experimental data are due to a limited temperature resolution.

This work was supported by the Spanish Ministry of Science and Innovation and EU FEDER (Project Nos. MAT 2007-65227 and MAT 2010-20537), the PAI of the Regional Government of Andalucia, and by the United States Office of Naval Research (Contract No. N0001410WX30490). R.C.F. acknowledges a research fellowship from the Regional Government of Andalucia.

${ }^{1}$ A. M. Tishin and Y. I. Spichkin, The Magnetocaloric Effect and Its Applications (Institute of Physics, Bristol, 2003).

${ }^{2}$ V. Franco, J. S. Blázquez, and A. Conde, Appl. Phys. Lett. 89, 222512 (2006).

${ }^{3}$ V. Franco, A. Conde, J. M. Romero-Enrique, and J. S. Blázquez, J. Phys.: Condens. Matter 20, 285207 (2008).

${ }^{4}$ V. Franco and A. Conde, Int. J. Refrig. 33, 465 (2010).

${ }^{5}$ M. Halder, S. M. Yusuf, M. D. Mukadam, and K. Shashikala, Phys. Rev. B 81, 174402 (2010).

${ }^{6}$ Yanyan Wang and Xiaofang Bi, Appl. Phys. Lett. 97, 022503 (2010).

${ }^{7}$ J. S. Kouvel and M. E. Fisher, Phys. Rev. 136, A1626 (1964).

${ }^{8}$ V. Franco, A. Conde, V. Provenzano, and R. D. Shull, J. Magn. Magn. Mater. 322, 218 (2010).

${ }^{9}$ S. K. Banerjee, Phys. Lett. 12, 16 (1964).

${ }^{10}$ C. M. Bonilla, J. Herrero-Albillos, F. Bartolomé, L. M. García, M. ParraBorderías, and V. Franco, Phys. Rev. B 81, 224424 (2010).

${ }^{11}$ K. A. Gschneidner, Jr. and V. K. Pecharsky, Annu. Rev. Mater. Sci. 30, 387 (2000).

${ }^{12}$ E. Brück, J. Phys D 38, R381 (2005).

${ }^{13}$ K. A. Gschneidner, Jr., V. K. Pecharsky, and A. O. Tsokol, Rep. Prog. Phys. 68, 1479 (2005).

${ }^{14}$ R. Caballero-Flores, V. Franco, A. Conde, K. E. Knipling, and M. A. Willard, Appl. Phys. Lett. 96,182506 (2010).

${ }^{15}$ V. Provenzano, A. J. Shapiro, and R. D. Shull, Nature (London) 429, 853 (2004).

${ }^{16}$ V. Franco, J. S. Blázquez, and A. Conde, J. Appl. Phys. 100, 064307 (2006).

${ }^{17}$ K. E. Knipling, M. Daniil, and M. A. Willard, Appl. Phys. Lett. 95, 222516 (2009).

${ }^{18}$ B. Widom, J. Chem. Phys. 43, 3898 (1965).

${ }^{19}$ R. B. Griffiths, Phys. Rev. 158, 176 (1967).

${ }^{20}$ V. Franco, A. Conde, M. D. Kuzmin, and J. M. Romero-Enrique, J. Appl. Phys. 105, 07A917 (2009).

${ }^{21}$ A. Arrott and J. E. Noakes, Phys. Rev. Lett. 19, 786 (1967).

${ }^{22}$ V. Franco, A. Conde, and L. F. Kiss, J. Appl. Phys. 104, 033903 (2008). 